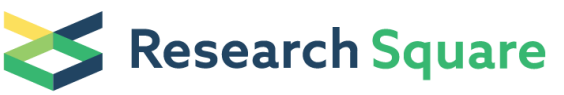 \\ Preprints are preliminary reports that have not undergone peer review. \\ They should not be considered conclusive, used to inform clinical practice, \\ or referenced by the media as validated information.
}

\section{Assessing Thailand's 1-3-7 surveillance strategy in accelerating malaria elimination}

Prayuth Sudathip

Division of Vector Borne Diseases, Department of Disease Control, Ministry of Public Health

\section{Sathapana Naowarat}

Inform Asia: USAID's Health Research Program, RTI International

\section{Suravadee Kitchakarn}

Division of Vector Borne Diseases, Department of Disease Control, Ministry of Public Health

\section{Deyer Gopinath}

World Health Organization

\section{Donal Bisanzio}

Inform Asia: USAID's Health Research Program, RTI International

\section{Niparueradee Pinyajeerapat}

U.S. President's Malaria Initiative, United States Agency for International Development (USAID), Regional Development Mission for Asia

\section{David Sintasath}

U.S. President's Malaria Initiative, United States Agency for International Development (USAID), Regional Development Mission for Asia

\section{Jui A. Shah ( $\nabla$ juishah@rti.org )}

Inform Asia: USAID's Health Research Program, RTI International

\section{Research Article}

Keywords: Elimination, surveillance, 1-3-7 strategy

Posted Date: February 8th, 2022

DOI: https://doi.org/10.21203/rs.3.rs-1326679/v1

License: (c) (1) This work is licensed under a Creative Commons Attribution 4.0 International License. Read Full License 


\section{Abstract}

Background: Thailand's strong malaria elimination program relies on effective implementation of its 1-3-7 surveillance strategy, which was endorsed and implemented nationwide in 2016. For each confirmed malaria patient, the Ministry of Public Health's Division of Vector Borne Diseases (DVBD) ensures completion of case notification within 1 day, case investigation within 3 days, and foci investigation within 7 days. To date, there has not been a comprehensive assessment of the performance and achievements of the 1-3-7 surveillance strategy although such results could help Thailand's future malaria elimination strategic planning.

Methods: This study examined adherence to the 1-3-7 protocols, tracked progress against set targets, and examined geographic variations in implementation of the 1-3-7 strategy in the program's initial five years. An auto-regressive integrated moving average (ARIMA) time series analysis with seasonal decomposition assessed the plausible implementation effect of the 1-3-7 strategy on malaria incidence in the program's initial five years. The quantitative analysis included all confirmed malaria cases from public health and non-governmental community facilities from October 2014 to September 2021 (fiscal year [FY] 2015 to FY 2021) $(n=77,405)$. The spatial analysis included active foci with known geocoordinates that reported more than five cases from FY 2018 to FY 2021.

Results: From FY 2017 to FY 2021, on-time case notification improved from $24.4 \%$ to $89.3 \%$, case investigations from $58.0 \%$ to $96.5 \%$, and foci investigations from $37.9 \%$ to $87.2 \%$. Adherence to timeliness protocols did not show statistically significant variation by area risk classification. However, adherence to 1-3-7 protocols showed a marked spatial heterogeneity among active foci, and the ARIMA model showed a statistically significant acceleration in the reduction of malaria incidence. The 1-3-7 strategy national indicators and targets in Thailand have shown progressive success, and most targets were achieved for FY 2021.

Conclusion: The results of Thailand's 1-3-7 surveillance strategy are associated with a decreased incidence in the period following the adoption of the strategy although there is notable geographic variation. The DVBD will continue to implement and adapt the 1-3-7 strategy to accelerate progress toward malaria elimination. This assessment may be useful for domestic strategic planning and to other countries considering more intensive case and foci investigation and response strategies.

\section{Background}

Thailand has a long history of conducting case investigations, case classifications, and focus investigation and response, as outlined in the Malaria Control Guidelines for Public Health Workers (1). Between 2012 and 2015, the Division of Vector Borne Diseases (DVBD) in Thailand's Ministry of Public Health (MOPH) reported significant reductions in the blood slide positivity rate to less than $5 \%$ among suspected cases with fever and in annual parasite incidence (API) to less than 1 per 1,000 population (range $0.38-0.82)(2,3)$. Reaching these milestones allowed the DVBD to transition from a malaria 
control program to a malaria elimination program; details of this transition are presented in Lertpiriyasuwat et al., 2021 (4). As part of the transition and based on results of a malaria program review in $2015(2,3)$, the MOPH emphasized its commitment to surveillance as a core intervention by introducing the 1-3-7 malaria surveillance strategy in 2016.

The 1-3-7 malaria surveillance strategy is adapted from China, where it is considered a key factor in the country's achievement of zero locally transmitted cases by 2017. The 1-3-7 strategy is operationalized with a simplified set of targets that outline responsibilities, actions, and the time frame for the crucial surveillance and response components of rapidly identifying infections and preventing them from spreading (2). The time frame is delineated as follows: within one day of diagnosis (i.e., 24 hours), local health staff are required to report the confirmed malaria case through the online Malaria Information System (MIS). Within three days, case investigation and classification must be completed to determine whether the case was locally acquired or imported. Finally, within seven days, a focus investigation and tailored response based on the case investigation results must be completed for each index case $(5,6)$.

The 1-3-7 strategy builds on Thailand's long-standing history of case notification, case classification, and focus investigation and response, with the addition of stricter time protocols to encourage adherence. Thailand's National Malaria Elimination Strategy (NMES) 2017-2026 includes annual targets to support monitoring and evaluation of each component of the strategy. These targets were approved by the Cabinet of the Royal Thai Government in 2016 and aim to reach zero indigenous malaria cases by 2024 (2).

Since fiscal year (FY) 2015, the baseline year for the 1-3-7 strategy, malaria incidence in Thailand has declined from 0.37 per 1,000 population to just 0.04 per 1,000 population in FY 2021. The DVBD has also verified 42 of its 77 provinces as malaria-free, suggesting that the malaria elimination program is progressing as intended. Remaining foci and high-transmission areas are concentrated in border provinces: Myanmar in the west, Cambodia in the east, and Malaysia in the South $(7,8)$. The movement of people across international borders is a main challenge that continues to thwart malaria elimination in the Greater Mekong Subregion (GMS); districts bordering Thailand often have different strategies in place and, as seen in Figure 1, may have higher malaria burden (9-12). High population mobility and a large workforce of migrant workers make it difficult to track patients over time to ensure radical cure in this region of high Plasmodium vivax prevalence $(6,13-16)$.

The DVBD has programmatically documented improvements in the timeliness of case reports and investigations $(3,4)$. However, there has not been an in-depth examination of the performance and achievements of the 1-3-7 surveillance strategy in accelerating malaria elimination in Thailand. Adopting the 2024 elimination goal and related milestones without monitoring progress may lead to wasted resources and missed opportunities to enhance efficiency. This study examines adherence to the 1-3-7 protocols outlined in the Guidelines for Malaria Elimination for Public Health Workers, tracks progress against set targets in the NMES, and examines geographic variation in implementation and results (17). This assessment of the strategy's first five years of implementation will help Thailand's future strategic 
planning and may be useful to other countries considering these more intensive case and foci investigation and response strategies.

\section{Data And Methods}

The analysis included all parasitologically confirmed cases (by microscopy or rapid diagnostic test) from public health and non-governmental community facilities reported from October 2014 to September 2021, representing FY 2015 to FY 2021. The study utilized FYs because Thailand's malaria program and database are based on FY targets. Quantitative malaria data were extracted from the national routine malaria information system, reviewed, and analyzed using IBM SPSS Statistics version 22. Spatial analyses were performed using R (18), Quantum GIS (19), and GeoDa (20). To ensure high quality data and meaningful results, the authors verified, cross-checked, and cleaned data; the final dataset is described in the Results section. The analysis was threefold: (1) examining adherence to 1-3-7 protocols, (2) determining achievement of results compared to nationally set targets, and (3) understanding geographic variations that could explain continued transmission patterns.

In line with the National Strategic Operational Plan 2017-2021, FY 2015 was treated as a baseline year, and FY 2017 was treated as the first year of full-coverage implementation, leaving FY 2016, as a "buffer year" in measurement analyses (21). The exclusion of FY 2016 accounted for the extraordinary effort that was required to develop, pilot test, and roll out materials and interventions to subnational officers to launch the 1-3-7 strategy. The buffer year also accommodated a policy change of stricter reactive case detection (RACD) inclusion criteria. Whereas RACD was previously conducted for 100 to 150 people (approximately 20 to 30 households) or a radius 1 to $2 \mathrm{~km}$, in FY 2017 a narrower screening of 50 people (approximately 10 households) within 1 to $2 \mathrm{~km}$ was adopted nationally based on reducing malaria burden. Spatial analyses were conducted for FY 2018-FY 2021 only, when 1-3-7 performance was more consistent.

Adherence to the 1-3-7 requirements was measured with descriptive statistics on malaria case notification, case investigation and classification, foci investigation and classification, and responses results, as outlined in the National Strategic Operational Plan (Table 1). Results were consolidated by month from FY 2017-FY 2021.

To assess achievement, values for key indicators were compared with national targets from the NMES. Values for supplementary indicators on RACD, for which the NMES does not specify annual targets (Table 1), were also reported to show progress over time. Outcome and impact indicators in the NMES track the number of villages and districts free from reported indigenous cases (Table 1). In Thailand, cases classified as "indigenous" refer to patients who contracted malaria in the village where they lived during the infection period. Any focus that has recorded an indigenous case in the previous three years (based on an annual focus classification cycle) is considered to be an "active focus" (22). It is worth noting that the number of active foci has decreased dramatically, from 2,227 in FY 2013 to 700 in FY 2019, as reported in Sudathip et al., and has further dropped to 469 in FY 2021 (22, 23). 
To assess the plausible effect of the 1-3-7 strategy's implementation on malaria incidence toward the elimination target of 2024, an auto regressive integrated moving average (ARIMA) was used to fit the time series model with trend and random components for active foci. Other key prevention and elimination strategies stayed the same over the study period, making this an appropriate approach to assess plausible results of the new strategy (23).

Thailand's 1-3-7 protocol is intended to be standardized across all subnational units. However, it is possible that the strategy's components are operationalized differently or that provincial teams face unique challenges, which could influence the strategy's effect (24). Spatial heterogeneity of adherence to 1-3-7 protocols was investigated among all active foci with known geocoordinates that reported more than five cases from FY 2018 to FY 2021. The threshold of five cases was set arbitrarily to avoid inclusion of foci with very few investigated cases. Adherence was measured using the following four indicators: percentage of cases reported within 1 day, percentage of cases investigated within 3 days, percentage of cases for which RACD was completed within 7 days, and percentage of cases with the full 1-3-7 sequence performed without delays. A hot spot analysis based on the $\mathrm{G}^{*}$ local spatial clustering test (24) was used to identify those areas with foci showing statistically significant lower adherence to the 1-3-7 schedules compared to all other foci. The significance of the results obtained by the $\mathrm{G}^{*}$ local spatial test was computed by comparing observed values to a random case distribution (null hypothesis) by randomly re-assigning the values of the tested indicator across the foci. The statistical significance calculation was based on 10,000 Monte Carlo randomizations ( $p<0.05$, with Bonferroni correction).

\section{Results}

Data from 77,405 malaria cases reported to the Malaria Information System (MIS) were split into 24,332 (31.4\%) cases for FY 2015; 17,578 (22.7\%) cases for the FY 2016 transition period; and 35,495 (45.9\%) cases for the FY 2017-FY 2021 post-intervention period. Adherence and achievement analyses included all cases with complete data on 1-3-7 indicators, which were available for 15,887 (90.4\%) of FY 2016 cases and 32,957 (92.9\%) of FY 2017-FY 2021 post-intervention cases; the remainder were not included for analysis due to missing or duplicate data. Recurrences were treated as new cases for the purpose of this study.

\section{Adherence to 1-3-7 protocols}

\section{Adherence to case notification within 1 day}

The monthly proportion of confirmed malaria cases reported within 24 hours to the system increased over the study period, from 18.2\% in October 2016 to 80.7\% in September 2021 (Figure 2). This increase was steady despite the fact that reporting rates vary based on malaria seasonality. During the peak transmission month of June, the proportion of timely notification increased steadily from $30.1 \%$ in FY $2017(567 / 1,881)$ to $92.6 \%$ in FY $2020(823 / 889)$ and 88.0\% in FY $2021(462 / 525)$. Note that there was a temporary drop of the timely notification proportion, to $56.2 \%$ at the end of FY 2019 , due to an MIS database security breach in August 2019 that temporarily disabled data entry. However, the DVBD was 
quick to respond to the issue, so malaria officers inputted data retrospectively, data entry was quickly resumed, and delays in reporting were limited to only two months (25).

Adherence to case investigation within 3 days

The monthly proportion of malaria cases investigated improved from $73.8 \%$ in October 2016 to $97.8 \%$ by September 2021, with substantial growth each year (Figure 3). The proportion of case investigations within the 3-day requirement also improved over time, from 52.4\% in June 2017 to $91.9 \%$ by September 2021 , even after seasonality is considered. The proportion of malaria cases investigated during the peak season in June rose from 64.1\% $(1,206 / 1,881)$ in FY 2017 to $97.3 \%(511 / 525)$ in FY 2021. During the low transmission season, case investigation followed the same upward trend, rising from $72.7 \%(421 / 579)$ in FY 2017 to 99.3\% (139/140) in FY 2021. Reporting timeliness also increased from $52.4 \%$ and $56.1 \%$ in the FY 2017 peak and low seasons, respectively, to $95.6 \%$ and $96.4 \%$ in the FY 2021 peak and low seasons, respectively.

Adherence to foci investigation and response within 7 days

The monthly proportion of RACD conducted among the cases that required RACD improved substantially over the study period, from 56.5\% in October 2016 to $83.2 \%$ by September 2021 (Figure 4). The proportion of RACD events conducted on time (within 7 days of case notification) also made dramatic improvement over the study period, from $48.2 \%$ to $80.3 \%$. These proportions did not vary much between high and low transmission seasons. However, adherence rates were higher in FY 2020 than FY 2021, likely due to COVID-related movement restrictions that compromised access to rural communities.

Comparison by area classification

Comparative t-tests showed that adherence to the DVBD's timeliness protocols did not show statistically significant variation by area classification. Before and during the buffer year of 1-3-7 implementation, timely case reporting was higher among active foci than among cleared foci with an index case $(p<$ 0.05). Although active foci continued to show more timely surveillance and response than cleared foci, in the post-intervention period, these differences were not statistically significant.

\section{Achievements of the 1-3-7 surveillance strategy compared to national targets}

The second part of this analysis compared actual results to targets laid out by FY, divided into output results and outcome/impact results (see Table 2 for summarized results). The NMES uses FY 2015 as a baseline year.

\section{Output indicators}

Overall, results on output indicators were strong. First, malaria cases reported in the MIS within the 1-day (or 24-hours) period showed consistent improvement over time, with rates reaching targets in FY 2019, FY 2020, and FY 2021 (77.1\%, 87.8\%, and 89.3\%, respectively). Second, the proportion of cases investigated 
within 3 days dramatically increased from baseline to $96.5 \%$, exceeding the FY 2021 target (95\%). Lastly, for foci investigation and response conducted within 7 days, the outputs met the targets in every FY, with the FY 2020 and FY 2021 proportions of $84.1 \%$ and $87.2 \%$ both surpassing the FY 2021 target.

The authors also examined the supplemental output indicators for which targets were not defined in the NMES, with results summarized in Table 3. During FY 2017-FY 2021, 14,277index cases triggered RACD (83.3\% of total cases eligible for RACD), leading to 866,920 people being screened: $1,513(0.17 \%)$ were positive for malaria infection. Each year, the number of individuals screened during RACD events dropped, with the proportional yield of positive cases also decreasing from $0.23 \%$ in FY 2017 to $0.11 \%$ in FY 2021 . RACD methods produced nearly equal yields for both active foci and cleared foci with a confirmed index case $(0.19 \%$ versus $0.16 \%)$.

\section{Outcome/impact indicators}

For the outcome indicator of percentage of districts without local transmission for at least three years (among a total of 928 districts in Thailand), targets were reached every FY between 2017 and 2019 (80\%, $83 \%$, and $85 \%$ respectively). Progress plateaued in FY 2020 and FY 2021, whereby district achievement reached $86 \%$ and $85 \%$, missing the $90 \%$ and $95 \%$ targets, respectively. The second outcome indicator is the number of villages with malaria transmission, which dropped from 2,310 in FY 2017 to 469 in FY 2021 (Table 2). This indicator has shown better-than-expected results, with substantial reductions beyond the set target each year, showing strides in interrupting community transmission.

These results were supplemented by other data collected by the DVBD on the population at risk in active foci, which from FY 2017 to FY 2021 dropped from 766,548 to 287,464 (Figure 5), and a decrease in the number of active foci from 2,310 to 469 for the same period. Because the DVBD's unit of analysis for daily assessments of routine data was the focus level, the program's MIS collated more granular geotemporal data on malaria-free area classification than what was required for NMES reporting.

\section{Observed and predicted trend in incidence}

The annual positive rates of all malaria species were used to establish an ARIMA model in active foci areas $(\mathrm{R} 2=0.72)$ (Figure 6). Prior to the 1-3-7 strategy's launch, malaria incidence among active foci was decreasing annually by 1.02 per 1,000 population at risk. After the launch in FY 2016, the ARIMA model showed an additional reduction to 1.31 cases per 1,000 population at risk per year. Each subsequent year has seen further reductions, averaging $1.36(p=0.62)$ annually, indicating an acceleration in the reduction of malaria incidence during the implementation period. The trend is forecasted to continue through FY 2024.

\section{Spatial analyses among active foci}

During the study period, active foci were clustered at Thailand's international borders. Adherence to 1-3-7 protocols showed a marked spatial heterogeneity among active foci (Figure 7), with southern and eastern foci reporting lowest adherence. Among the indicators examined, timely case investigation showed the 
highest results and lowest spatial heterogeneity (Figure 7); however, some foci in high-burden areas in the northwest showed poor adherence $(p<0.05)$. The $\mathrm{G}^{*}$ test identified foci with significantly $(p<0.05)$ lower adherence to overall 1-3-7 timeliness, mostly in Sisaket province in the east, Kanchanaburi and Ratchaburi provinces in the west, and Yala province in the south. (Figure 8).

\section{Discussion}

The 1-3-7 strategy is an innovative surveillance and response intervention for accelerating progress toward Thailand's malaria elimination goal by 2024. Even if only one malaria case is detected, local health personnel must respond immediately per standard operating procedures, making the strategy quite sensitive to epidemiological changes $(3,4)$. Adherence rates show that the protocols are being successfully implemented, with case notifications within 1 day increasing from $24.4 \%$ to $89.3 \%$, case investigations within 3 days increasing from $58.0 \%$ to $96.5 \%$, and foci investigations and response within 7 days increasing from $37.9 \%$ to $87.2 \%$ during the first five years of implementation.

Data quality is high, particularly in terms of completeness and timeliness; in fact, the DVBD's coordination and communication across subnational units has been cited as a success factor for the 1-3-7 strategy (3, 4). The national team provides close operational support to subnational teams on data quality and compliance with 1-3-7 protocols. The DVBD regularly monitors for unusual data, such as an increase in cases compared to historic data or a case identified in a province already verified as malaria free. The program immediately and directly communicates queries to specific subnational officers via its malaria group mobile chat application with more than $\mathbf{4 0 0}$ malaria members from across the country from every level of the health system. Subnational officers respond publicly, describing investigation efforts, providing contextual information and photographs, and following up to share subsequent results or requesting further guidance. The DVBD also distributes a monthly report collating unusual events and data trends to all provincial offices, in addition to a monthly report tracking progress on national indicators to leadership in the Department of Disease Control.

To ensure continued progress toward the ultimate goal of zero malaria, the DVBD is conducting further analyses on each component of the 1-3-7 strategy to better understand reasons behind the documented geographic variation. Thailand's routine surveillance data show that high-incidence provinces have many more active foci per province and more cases per focus. However, the provinces with low adherence identified by the spatial analysis have unique challenges related to drug efficacy (Sisaket in the east), migration patterns (Kanchanaburi and Ratchaburi in the west), and civic unrest (Yala in the south). Assessing areas of persistent transmission can help target resources to accelerate elimination, so the DVBD is currently developing a comprehensive statistical model that includes 1-3-7 implementation, environmental factors, and social and behavioral characteristics.

The 1-3-7 strategy national indicators and targets in Thailand have shown progressive success-more than $80 \%$ achievement in every component. Most targets were achieved for FY 2021, suggesting that the DVBD set out ambitious but realistic goals in the NMES. Because few other changes were implemented in 
the malaria control program at the time of the 1-3-7 adoption, the results of this study suggest it is plausible that the strategy is associated with the documented $80 \%$ decline in malaria burden from $\mathrm{FY}$ 2017 to FY 2021 and the notable increase in malaria-free foci and districts.

Of course, the context also evolves, particularly as the protracted COVID-19 pandemic poses a serious additional challenge to global progress toward malaria elimination (26). In January 2020, Thailand became the second country in the world to confirm a COVID-19 case. Several protective movement restrictions were implemented in the subsequent months, resulting in just 3,553 cases of COVID-19 recorded in FY 2020 (27). These cases were mostly limited to urban areas, contrary to malaria cases that are concentrated in forested border areas, so there was limited overlap in epidemiology. However, in FY 2021, over 1.6 million cases were reported as the epidemiology widened to affect most areas of the country (27). COVID-19 cases peaked during the high malaria transmission period from July to September and may have affected malaria interventions by overwhelming health systems, altering population movement and health seeking behaviors, and diverging resources and frontline workers. These disruptions could have affected 1-3-7 implementation and results, thereby meriting further analyses.

Thailand's declining malaria burden supports its collaborative regional goals and programs. Since 2000, GMS countries have successfully reduced the reported number of all malaria species cases by $90 \%$ and Plasmodium falciparum ( $P f$ ) cases by $97 \%(26)$. This is coupled with a decline in malaria deaths from 6,000 in 2000 to 10 in 2020 (28) in the GMS. Altogether, this is remarkable progress toward the region's collective goal of malaria elimination by 2030. Following China and Thailand, Myanmar, Cambodia, and Lao PDR began piloting the 1-3-7 strategy $(29,30)$. Continuation of the close partnership among GMS countries will help support regional goals and address some of the most challenging border hot spots identified by the spatial analysis. A starting point for expanded programming could be cross-border notification and foci response, utilizing the networks of community health workers that are acutely familiar with local populations, and providing additional training to boost 1-3-7 adherence in those areas.

\section{Limitations}

This assessment is not a true impact evaluation of the malaria elimination program or the 1-3-7 surveillance intervention of Thailand. There is substantial guidance on conducting robust impact evaluations for full-scale malaria interventions; however, these models have been designed for contexts with substantial malaria burden (30-32). Even the guidance issued for lower transmission settings is designed for API that is higher than those in Thailand (33). The ARIMA model is based on case burden and is a useful method to assess the 1-3-7 strategy's plausible effect on incidence. However, the model does not account for important programmatic and contextual factors.

This study used robust routine data from Thailand's national MIS (32). Data completeness, as reported in the results section, is less than $100 \%$, which is normal for routine data but requires appropriate interpretation. The cases not captured in the MIS are likely to be patients that have a deliberate reason to avoid the surveillance system or the formal public health system, and it is difficult to assess how many 
cases may be missed. Furthermore, response data are particularly challenging to interpret. RACD results are reported in aggregate at the foci level so coverage up to the required 50 individuals, 10 households, or $2 \mathrm{~km}$ is difficult to ascertain, as are case-level geolocation or demographics. The DVBD and its partners are working to ensure that the MIS is a complete and comprehensive source of malaria data before the 2024 elimination target.

\section{Next steps and road to elimination}

Although the 1-3-7 strategy has been successfully implemented and results have shown steady improvements, efforts must be sustained to address emerging challenges due to COVID-19, migration patterns, or sociopolitical dynamics in the $\operatorname{GMS}(7,16,34)$. Preventing resurgence and reintroduction of malaria are also growing priorities for the DVBD, based on lessons learned elsewhere $(35,36)$. These investments in malaria surveillance can enhance Thailand's health security and preparedness in crucial areas such as rapid detection, alert, and response, thereby building resilience against future infectious disease threats.

The DVBD has been intensifying its strategies to ensure appropriate surveillance for very lowtransmission settings $(37,38)$. Such strategies include enhancements to foci response and active surveillance protocols, advocacy for policy legislation, resources for additional staff trainings on accurate case management, support for improved data recording and reporting, and involvement of the private sector and military to ensure that the surveillance system is capturing all cases $(39,40)$. Effectively reaching marginalized populations may require innovative solutions, particularly as malaria burden and risk perception falls $(5,41)$.

\section{Conclusions}

The encouraging results of Thailand's 1-3-7 surveillance strategy are in line with the dramatically decreased incidence seen in the time period following the strategy's adoption. The malaria program will continue its emphasis on implementing and applying Thailand's 1-3-7 strategy, perhaps with additionally tailored activities for varying zones of transmission or that capitalize on community health networks and cross-border collaboration. The 1-3-7 strategy supports the DVBD's goals to achieve and sustain malaria elimination and could be a useful example for other countries in the GMS aiming to eliminate malaria.

\section{Abbreviations}

ARIMA auto-regressive integrated moving average

COVID-19 coronavirus disease 2019

DVBD Division of Vector Borne Diseases

FY fiscal year 
GMS Greater Mekong Subregion

MIS Malaria Information System

$\mathrm{MOPH} \quad$ Ministry of Public Health

NMES National Malaria Elimination Strategy

PMI President's Malaria Initiative

RACD reactive case detection

USAID United States Agency for International Development

\section{Declarations}

Ethics approval and consent to participate

Not applicable.

\section{Consent for publication}

Not applicable.

\section{Availability of data and materials}

The visualizations supporting the conclusions of this article are available in the Malaria Online repository, http://malaria.ddc.moph.go.th/.

\section{Competing interests}

The authors declare that they have no competing interests.

\section{Funding}

This study was made possible by the generous support of the American people through the U.S. President's Malaria Initiative (PMI) and United States Agency for International Development (USAID), under the terms of Cooperative Agreement AID-486-LA-15-00002 for Inform Asia: USAID's Health Research Program. The contents of this article are the responsibility of the authors and do not necessarily reflect the views of USAID, PMI, or the U.S. Government.

\section{Authors' contributions}

PS, DG, DS, and JAS conceptualized the manuscript. SN analyzed the quantitative results and DB analyzed the spatial results. SN and JAS interpreted the results and led the writing of the manuscript. All 
authors, including SK and NP, provided input and review. All authors read and approved the final manuscript.

\section{Acknowledgements}

The authors recognize all local public health teams and partners who have contributed to planning and implementing the 1-3-7 strategy at the national and field levels under the leadership of Thailand's Division of Vector Borne Diseases, Department of Disease Control, Ministry of Public Health.

\section{References}

1. Bureau of Vector Borne Disease. Guidelines of Malaria Control Practices for Public Health Workers. In: Control DoD, editor. MOPH: Division of Malaria Disease; 2009.

2. Bureau of Vector Borne Disease. National Malaria Elimination Strategy, Thailand 2017-2026. In: Control DoD, editor. Ministry of Public Health2016.

3. World Health Organization. Programmatic Review of the National Malaria Programme in Thailand: 31st August - 11th September 2015, Summary Report. 2016. Available from: https://apps.who.int/iris/bitstream/handle/10665/253958/9789290225133-eng.pdf?ua=1.

4. Lertpiriyasuwat C, Sudathip P, Kitchakarn S, Areechokchai D, Naowarat S, Shah JA, et al. Implementation and success factors from Thailand's 1-3-7 surveillance strategy for malaria elimination. Malaria J. 2021;20(201).

5. Lu G, Liu Y, Beiersmann C, Feng Y, Cao J, Müller O. Challenges in and lessons learned during the implementation of the 1-3-7 malaria surveillance and response strategy in China: a qualitative study. Infect Dis Poverty. 2016;5(94):1-11.

6. Feng J, Liu J, Feng X, Zhang L, Xiao H, Xia Z. Towards malaria elimination: monitoring and evaluation of the "1-3-7" approach at the China-Myanmar border. Am J Trop Med Hyg. 2016;95(4):806-10.

7. Sudathip P, Kongkasuriyachai D, Stelmach R, Bisanzio D, Sine J, Sawang S, and et al. The investment case for malaria elimination in Thailand: A cost-benefit analysis. Am J Trop Med Hyg. 2019;100:1445-53.

8. Tipmontree R, Fungladda W, Kaewkungwal J, Tempongko S, Schelp F. Migrants and malaria risk factors: A study of the Thai-Myanmar border. The Southeast Asian journal of tropical medicine and public health. 2009;40:1148-57.

9. Wangdi K, Gatton ML, Kelly GC, Clements AC. Cross-border malaria: A major obstacle for malaria elimination. Advances in Parasitology. 2015;89:79-107.

10. Wangdi K, Clements CAA. Ending Malaria Transmission in the Asia Pacific Malaria Elimination Network (APMEN) Countries: Challenges and the Way Forward. 2018. In: Towards Malaria Elimination - A Leap Forward [Internet]. [201-32]. Available from: http://dx.doi.org/10.5772/intechopen.75405. 
11. Hlongwana $\mathrm{K}$, Tsoka-Gwegweni J. Towards the implementation of malaria elimination policy in South Africa: The stakeholders' perspectives. Global Health Action. 2017;10(1288954).

12. Al Zahrani $H$, Omar IA, Abdoon MOA, Ibrahim AA, Alhogail A, Elmubarak $M$, and et al. Cross-border movement, economic development and malaria elimination in the Kingdom of Saudi Arabia. BMC Medicine. 2018;16(98).

13. Wangdi K, Banwell C, Gatton ML, Kelly GC, Namgay R, Clements AC. Development and evaluation of a spatial decision support system for malaria elimination in Bhutan. Malaria J. 2016;15(180).

14. Huang F, Zhang L, Xue J, Zhou N, Thi A, Zhang J, and et al. From control to elimination: a spatial-temporal analysis of malaria along the China-Myanmar border. Infect Dis Poverty. 2020;9(158):1-13.

15. Delacollette C, D'Souza C, Christophel E, Thimasarn K, Abdur R, Bell D, and et al. Malaria trends and challenges in the Greater Mekong Subregion. Southeast Asian J Trop Med Public Health. 2009;40(4):674-91.

16. Cui L, Yan G, Sattabongkot J, Cao Y, Chen B, Chen X, and et al. Malaria in the Greater Mekong Subregion: heterogeneity and complexity. Acta Trop. 2012;121:227-39.

17. Bureau of Vector Borne Disease. Malaria Elimination Guideline for District and Sub-district Health Worker. In: Control DoD, editor. Ministry of Public Health2019.

18. R Core Team. R: A language and environment for statistical computing. Vienna, Austria: $R$ Foundation for Statistical Computing; 2021. Available from: https://www.R-project.org/.

19. QGIS Development Team. QGIS Geographic Information System, . Open Source Geospatial Foundation Project; 2021. Available from: http://qgis.osgeo.org.

20. Luc A, Syabri I, Kho Y, . An Introduction to Spatial Data Analysis. Geographical Analysis. GeoDa:. 2006;38(1):5-22.

21. Bureau of Vector Borne Disease. National Strategic Operation Plan, Thailand 2017-2021. In: Control DoD, editor. Ministry of Public Health2016.

22. Sudathip P, Kitchakarn S, Shah JA, Bisanzio D, Young F, Gopinath D, et al. A foci cohort analysis to monitor successful and persistent foci under Thailand's Malaria Elimination Strategy. Malaria J. 2021;20(1):118.

23. Fretheim A, Zhang F, Ross-Degnan D, Oxman AD, Cheyne H, Foy R, et al. A reanalysis of cluster randomized trials showed interrupted time-series studies were valuable in health system evaluation. J Clin Epidemiol. 2015;68:324-33.

24. Ord J, Getis A. Local spatial autocorrelation statistics. Distributional issues and an application Geographical Analysis 1995;27:286-306.

25. Division of Vector Borne Disease. Malaria Online: The Digital Surveillance System for Thailand Malaria Elimination. In: Department of Disease Control, editor. MOPH2019.

26. World Health Organization. World malaria report 2020: 20 years of global progress and challenges2020. Available from: https://www.who.int/teams/global-malaria- 
programme/reports/world-malaria-report-2020.

27. Digital Government Development Agency (DGA). Daily report for COVID-19, Thailand dataset, 2021 Date accessed: 21 June 2021. Available from: https://data.go.th/dataset/covid-19-daily.

28. Mekong Malaria Elimination Programme. Countries of the Greater Mekong ready for the "last mile" of malaria elimination2020. Available from: https://www.who.int/publications-detail-redirect/WHO-UCNGMP-MME-2020.05.

29. Aung $P$, Thein WZ, Hein NMZ, Aung TK, Mon ON, Linn YYN, and et al. Challenges in early phase of implementing the 1-3-7 surveillance and response approach in malaria elimination setting: A field study from Myanmar. Infect Dis Poverty. 2020;9(18).

30. Kheang S, Sovannaroth S, Barat LM, Dysoley L, Kapella BK, Po L, et al. Malaria elimination using the 1-3-7 approach: lessons from Sampov Loun, Cambodia. BMC Public Health. 2020;20(544).

31. Hershey C, Bhattarai A, Florey LS, McElroy PD, Nielsen CF, Ye Y, et al. Implementing impact evaluations of malaria control interventions: Process, lessons learned, and recommendations. Am J Trop Med Hyg. 2017;97(3_Suppl):20-31.

32. Rowe A, Steketee RW, Arnold F, Wardlaw T, Basu S, Bakyaita N, et al. Viewpoint: evaluating the impact of malaria control efforts on mortality in sub-Saharan Africa. trop Med Int Health. 2007;12(12):152439.

33. Ashton A, Prosnitz D, Andrada A, Herrera S, Ye Y. Evaluating malaria programmes in moderate- and low-transmission settings: practical ways to generate robust evidence. Malaria J. 2020;19(75):1-14.

34. Geng J, Malla P, Zhang J, Xu S, Li C, Zhao Y, and et al. Increasing trends of malaria in a border area of the Greater Mekong Subregion. Malaria Journal. 2019;18(309).

35. Premaratne R, Wickremasinghe R, Ranaweera D, Gunasekera WKA, Hevawitharana M, Pieris L, Fernando D, Mendis K. Technical and operational underpinnings of malaria elimination from Sri Lanka. Malaria J. 2019;18(256).

36. Nkumama N, O'Meara PW, Osier HAF. Changes in Malaria Epidemiology in Africa and New Challenges for Elimination. Trends in Parasitology. 2017;33(2):128-40.

37. World Health Organization. An operation manual: disease surveillance for malaria control 2012 [December 11, 2020]. Available from: https://www.paho.org/hq/dmdocuments/2012/WHO-DiseaseSurveillance-Malaria-Control-2012.pdf.

38. Hay SI SD, Snow RW. Measuring malaria endemicity from intense to interrupted transmission. Lancet Infectious Diseases 2008;8:369-78.

39. World Health Organization. Malaria surveillance, monitoring \& evaluation: a reference manual.2018. Available from: https://apps.who.int/iris/bitstream/handle/10665/272284/9789241565578eng.pdf?ua=1.

40. Roh ME, Lausatianragit K, Chaitaveep N, Jongsakul K, Sudathip P, Raseebut C, et al. Civilian-military malaria outbreak response in Thailand: an example of multi-stakeholder engagement for malaria elimination. Malaria Journal. 2021;20(1):458. 
41. Bureau of Vector Borne Disease. Guide to malaria elimination for Thailand's Local Administrative Organizations and the health network. In: Department of Disease Control, editor.: Ministry of Public Health; 2019.

\section{Tables}

Table 1. 1-3-7 strategy's indicators and outputs and NMES indicators

\section{1-3-7 Key and Supplemental Indicators}

Day - Total malaria cases reported to the database

1

- Number and percentage of malaria cases reported to the malaria database within 1 day

Day $\quad$ Number and percentage of malaria cases reported to the malaria database that were 3 investigated

- Number and percentage of case investigations completed within 3 days

Day - Number of cases reported to the malaria database that require RACD screening 7

- Number and percentage of RACD events conducted

- Number and percentage of RACD events conducted within 7 days

Supplementary indicators without set targets

- Number of individuals targeted for RACD screening

- Number and percentage of individuals screened during RACD

- Number and percentage of positive cases newly detected during RACD

\section{NMES Outcome and Impact Indicators}

- Percentage of districts with no indigenous malaria case transmission for at least 3 years

- Number of villages with indigenous malaria cases transmission

Table 2. Achievement of 1-3-7 strategy, national indicators, and targets for FY 2017-FY 2020 
Indicators

OUTPUT INDICATORS

\section{Percentage of malaria cases reported in the MIS within 24 hours}

Total number of malaria cases reported to the database

Number of malaria cases reported to the malaria database within 1 day

\section{Percentage of malaria cases investigated within 3 days}

Number of malaria cases reported to the malaria database

Number of malaria cases reported to the malaria database that were investigated

Number of case investigations completed within 3 days

\section{Percentage of malaria cases investigated with foci response conducted within 7 days}

Number of cases reported to the malaria database that require RACD screening

Number of RACD events conducted

Number of RACD events conducted within 7 days

\section{OUTCOME AND IMPACT} INDICATORS

Number and percentage of districts with no indigenous malaria case

transmission for at least 3 years $(N=928)$

$\begin{array}{llllll}\text { FY } & \text { FY } & \text { FY } & \text { FY } & \text { FY } & \text { FY } \\ 2015 & 2017 & 2018 & 2019 & 2020 & 2021\end{array}$

Target

Achievement $\quad 18.0 \%$ 24,33
$50 \%$

$24.4 \%$

14,954

7,368

5,845

4,421

2,835

4,383

3,643

4,388

4,508

3,881

2,531

Target

Achievement

$85 \%$

$90 \%$

$95 \%$

$95 \%$

$95 \%$

$44.1 \%$

$58.0 \%$

$70.4 \%$

$84.0 \%$

$94.7 \%$

$96.5 \%$

24,332

14,954

7,368

5,845

4,421

2,835

$15,992 \quad 10,614$

6,140

5,532

4,331

2,785

Target

$30 \%$

$50 \%$

$60 \%$

$70 \%$

$80 \%$

$35.7 \%$

$37.9 \%$

$55.2 \%$

$75.3 \%$

$84.1 \%$

$87.2 \%$

7,704

5,848

3,566

3,225

2,557

1,936

$\begin{array}{llllll}7,704 & 5,848 & 3,566 & 3,225 & 2,557 & 1,936\end{array}$

\begin{tabular}{rrrrrr}
5,410 & 4,119 & 2,973 & 2,928 & 2,433 & 1,824 \\
\hline 2,750 & 2,214 & 1,969 & 2,428 & 2,150 & 1,689
\end{tabular}

Target

Achievement
696743

(75\%)

632

$748 \quad 767$

(80\%)

789

(85\%)

836

(90\%)

882

(95\%)

788

798

(83\%)

(85\%)

(86\%) 
Number of villages with indigenous malaria case transmission
Target

3,610

Achievement

5,552

2,310

2,707

1,895

1,232

739

1,676

1,382

1,187

469

Table 3. Performance of RACD events conducted among foci investigations for FY 2017-FY 2021

\begin{tabular}{|c|c|c|c|c|c|}
\hline \multirow[b]{2}{*}{ Indicator } & \multicolumn{5}{|c|}{ Achievement } \\
\hline & 2017 & 2018 & 2019 & 2020 & 2021 \\
\hline $\begin{array}{l}\text { Number of individuals screened during } \\
\text { RACD }\end{array}$ & 222,991 & 182,275 & 176,162 & 159,892 & 125,600 \\
\hline $\begin{array}{l}\text { Number of positive cases newly identified } \\
\text { using RACD }\end{array}$ & 507 & 311 & 314 & 248 & 133 \\
\hline Percent found positive from RACD events & $0.23 \%$ & $0.17 \%$ & $0.18 \%$ & $0.16 \%$ & $0.11 \%$ \\
\hline
\end{tabular}

\section{Figures}

API/1000 Population

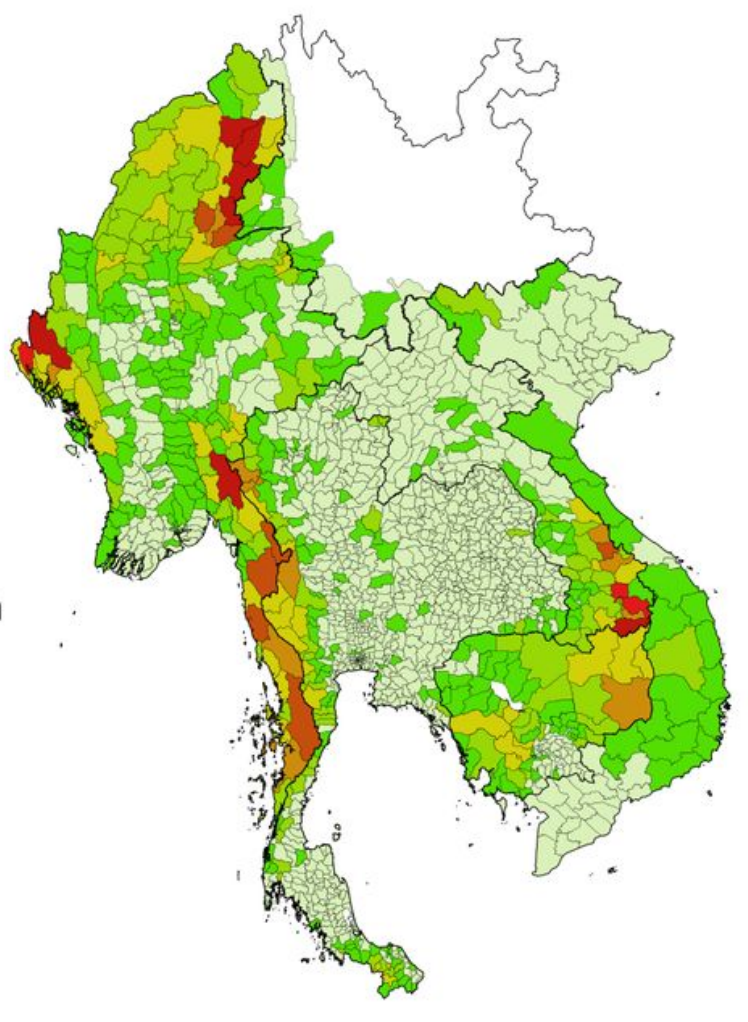

Figure 1

Malaria annual parasite incidence in GMS, FY 2021 
Source: Malaria Elimination Database, Mekong Malaria Elimination Programme, 2021 private communication

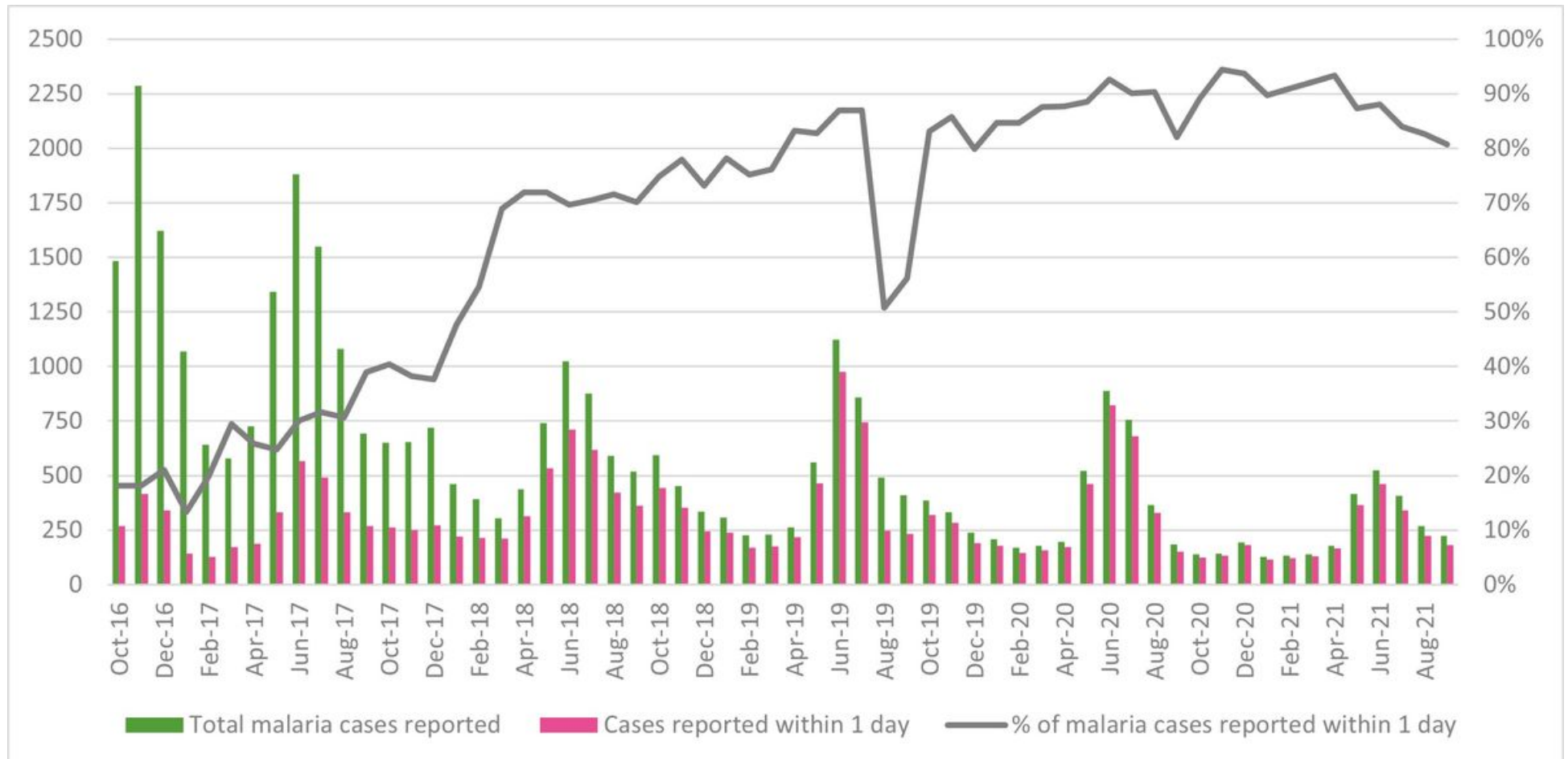

Figure 2

Number and percentage of malaria cases reported within 1 day, by month, FY 2017-FY 2021

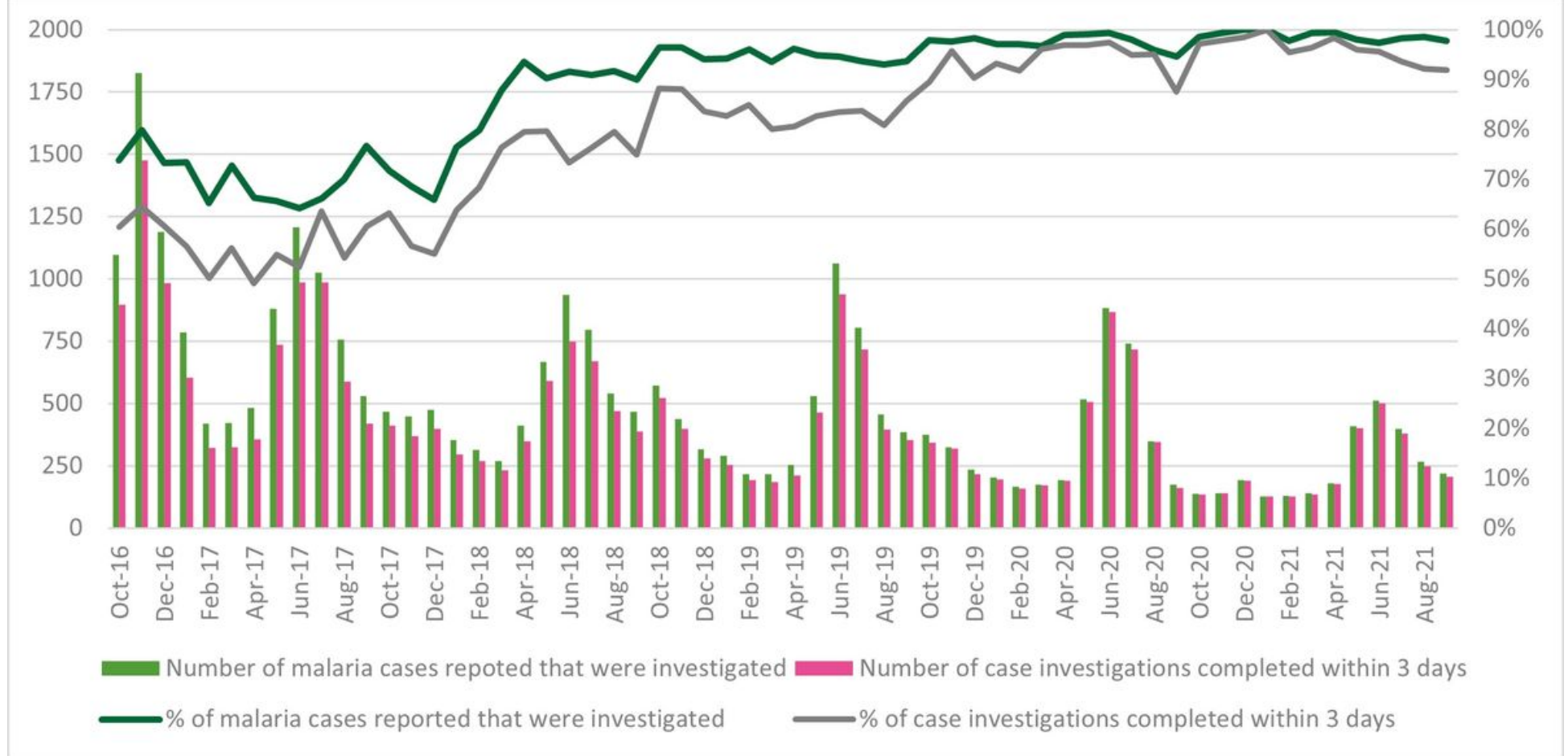

Figure 3 
Number and percentage of malaria cases investigated within 3 days, by month, FY 2017-FY 2021

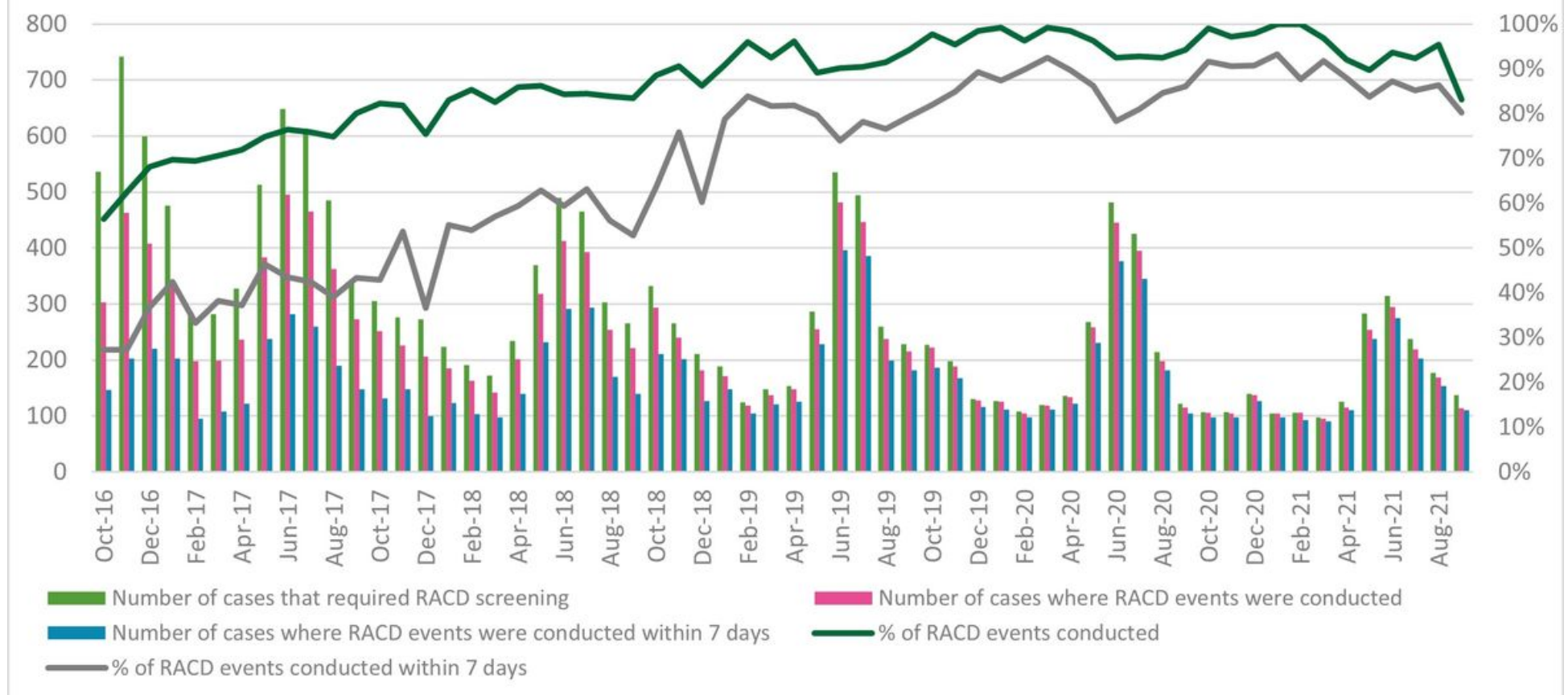

Figure 4

Number and percentage of foci investigated and responded to within 7 days, FY 2017-FY 2021

\begin{tabular}{|c|c|c|c|}
\hline Unit & Category & FY 2017 & FY 2021 \\
\hline \multirow{4}{*}{ District } & Indigenous & 119 districts (12.8\%) & 64 districts (6.9\%) \\
\cline { 2 - 4 } & Malaria-free = 1 year & 29 districts (3.1\%) & 22 districts (2.4\%) \\
\cline { 2 - 4 } & Malaria-free = 2 years & 32 districts (3.4\%) & 29 districts (3.1\%) \\
\cline { 2 - 4 } & Malaria-free $\geq 3$ years & 748 districts $(80.6 \%)$ & 813 districts $(87.6 \%)$ \\
\hline \multirow{2}{*}{ Focus } & Active foci & 2,310 & 469 \\
\cline { 2 - 4 } & Population at risk & 766,548 & 287,464 \\
\hline
\end{tabular}

Figure 5

District-level improvements in malaria free status from FY 2017 and FY 2021

Source: DVBD, MOPH, 2021 private communication

Figure 6

Observed and forecast malaria incidence per 1,000 population at risk in active foci areas FY 2015-FY 2024 
Figure 7

Spatial pattern of adherence to 1-3-7 protocols among active foci in Thailand, FY 2018-FY 2021

\section{Figure 8}

Active foci with significantly lower adherence to 1-3-7 protocols, FY 2018-FY 2021 The red dots indicate significant hot spots $(p<0.05)$ of low adherence to 1-3-7 timing identified by the $G *$ local spatial test. 